Research Article

\title{
Beam Tracking in Switched-Beam Antenna System for V2V Communication
}

\author{
Settawit Poochaya and Peerapong Uthansakul \\ School of Telecommunication Engineering, Suranaree University of Technology, Nakhon Ratchasima 30000, Thailand \\ Correspondence should be addressed to Peerapong Uthansakul; uthansakul@sut.ac.th
}

Received 30 October 2015; Revised 15 January 2016; Accepted 21 January 2016

Academic Editor: Lei Yu

Copyright (c) 2016 S. Poochaya and P. Uthansakul. This is an open access article distributed under the Creative Commons Attribution License, which permits unrestricted use, distribution, and reproduction in any medium, provided the original work is properly cited.

\begin{abstract}
This paper presents the proposed switched beam antenna system for $\mathrm{V} 2 \mathrm{~V}$ communication including optimum antenna half power beamwidth determination in urban road environments. SQP optimization method is selected for the computation of optimum antenna half power beamwidth. In addition, beam tracking algorithm is applied to guarantee the best beam selection with maximum RSSI. The results present the success of the proposed system with the increasing of V2V performance metrics. Also, V2V data dissemination via the proposed system introduces the enhancement of V2V link in terms of RSSI, PER, BER, $T_{\text {safe }}$, and $R_{\text {safe }}$. The results indicate the improvement of V2V link reliability. Consequently, the road safety is improved.
\end{abstract}

\section{Introduction and Related Works}

The increasing of vehicles in the world promotes the traffic congestions and accidents on the road. The works in [1-3] have presented that the intelligence of conventional transportation system is made by Dedicated Short Range Communication (DSRC) technology which provides a fast data transmission and a high reliability of the communication link. ITS/DSRC communication regime operates under IEEE802.11p (WAVE-DSRC) standard at $5.9 \mathrm{GHz}(7$ channels) working for different manners. ITS improves the road safety by sending useful information to the drivers on the road for preparing for a bad situation. There are two differences of data dissemination procedures which are categorized into $\mathrm{V} 2 \mathrm{~V}$ (Vehicle to Vehicle) communication and V2I (Vehicle to Infrastructure) communication. The work in [4] has introduced that the equipment for ITS/DSRC is OBU (On Board Unit) and RSU (Road Side Unit). Both devices are equipped with omnidirectional antenna. The works presented in $[5,6]$ have introduced that ITS/DSRC applications are separated into two major types: safety applications and nonsafety applications. Safety applications require for more link reliability and lower latency. The work in $[7,8]$ has presented that the impacts of accident on expressways rely on the decision of drivers. There are 2194 accidents occurring on the expressways. Also, the human lives lost and the costs of damaging are the significant issues which motivate the governments in the world to search for better solutions. There are many well known safety applications in the world such as collision warning system, road side alert, and Road Hazard Warning. However, the works in $[9,10]$ have presented that the phenomenon of transmitting signal for all directions introduces the signal power losing in undesired directions. As a result, a signal quality at the receiving side is not good enough. Also, the signal quality drops at the receiving vehicles. The works in [11, 12] have presented that an antenna having directional pattern is the solution. This is because an antenna having directional pattern can radiate the signal to the desired directions. The signal quality such as RSSI performance is improved. However, there are some limitations of an antenna having directional pattern such as hidden node problem and sometime cannot be used in some applications. The works in [13-17] indicated the powerful antenna technology which provided the reduction of interference effect and increasing the signal quality at the receiving vehicle. Smart antenna has been proposed for a solution. There are two types of smart antenna technology including a switched-beam antenna and an adaptive array antenna. The adaptive array antenna requires more 
processing times due to feedback control bits for adjusting the phase and amplitude of the transmitting signal. Switchedbeam system does not require complexity and intelligent methodology. Also, the processing time of switched-beam system is less than adaptive array system. However, the works presented in $[18,19]$ have introduced other significant factors to the performance of switched-beam system. One of these factors is the antenna half power beamwidth (HPBW). However, a conventional switched-beam system has an equal HPBW for each beam. The equal HPBW is determined without the consideration of vehicle positions on the road and the environments of the road so this causes the inefficiency of using conventional HPBW for V2V applications. Moreover, the improper HPBW indicate the chance of connection loss, low communication range, and low signal quality. The optimum HPBW will improve a signal quality at the receiving side. Moreover, to ensure that there is no connection loss until the data dissemination between vehicles, beam tracking algorithm has been proposed. In literatures, there are many methods of beam tracking algorithm. Focusing on the main idea of $\mathrm{V} 2 \mathrm{~V}$ communication, DSRC V2V requires fast and more reliability of the communication link. Then, the simplest beam tracking mechanism for V2V communication has been selected according to the works presented in [2023]. Low connection loss and simplest procedures are the advantage of RSSI-based beam tracking mechanism. Focusing on V2V DSRC communication, beam tracking provides a seamless connection of the radio link between vehicles. The vehicles can travel from one position to other positions on the road if the connected beam is suitable for the position of the vehicle on the road. Then the signal quality can be improved. As a result, the high radio link reliability with low connection loss and seamless connection will promote the road safety. Because the vehicle connects to $\mathrm{V} 2 \mathrm{~V}$ network all the time, vehicles inside a communication zone can receive a warning message in order to prepare for a bad situation on the road.

This paper employs the corner reflector type for antennas according to [24] because it is the simplest way and less complexity of antenna HPBW beam shaping technique. The details to apply 4-beam SWB system with optimum HPBW for V2V communication have been given later. This paper presents the experiments of the proposed system for the real urban road. The performance matrices in this paper are the same as presented in $[25,26]$ including RSSI, ECR $\left(R_{\text {safe }}\right)$, and TTC $\left(T_{\text {safe }}\right)$. Note that the speed limit in urban road [27] following the law is $60 \mathrm{~km} / \mathrm{hr}$. The results confirm that applying switched-beam antenna with optimum HPBW and beam tracking can enhance V2V communication link. Moreover, the results indicate the improvement of traffic safety for $74 \%$ when comparing to the conventional V2V communication system. Moreover, the method to find the optimum HPBW is firstly introduced in this paper by using SQP optimization method. The consideration of antenna half power HPBW can be separated into two planes, elevation and azimuth planes. Focusing on urban road area, a distance between vehicles is short. Then, the effect from azimuth HPBW is more than elevation plane. Thus, this paper focuses on the determination of optimum HPBW on only azimuth plane. Also, the results in terms of RSSI, BER, PER, and $R_{\text {safe }}$ and $T_{\text {safe }}$ have been examined through simulations and experiment. The contributions of this work can be addressed in the following:

(1) V2V Communication link reliability is increased due to the proposed system. The increasing of RSSI presents the improving of communication link performance metrics and link reliability such as BER and throughput. Low BER introduces a high reliability of $\mathrm{V} 2 \mathrm{~V}$ communication link. As a result, a receiving vehicle can receive the correct warning messages. Then, the chance of accident is indirectly reduced by the proposed system.

(2) $R_{\text {safe }}$ : RSSI enhancement introduces the increasing of coverage range of $\mathrm{V} 2 \mathrm{~V}$ message communication. The transmitting vehicle can communicate with receiving vehicle at a long distance. So, the vehicles receive the message at a long range which means that the drivers have more time to prepare for the bad situation on the road such as accidents and chain collisions. Thus, the outcome of the proposed system enhances the safety for the drivers. As a result, the proposed system will support the requirements of V2V DSRC safety applications.

(3) $T_{\text {safe }}$ : this parameter indicates the preparing time for a bad situation on the road. In addition, $T_{\text {safe }}$ refers to the collision time. The proposed system produces more $T_{\text {safe }}$. Also, the traffic safety has been improved due to the increasing of $T_{\text {safe }}$.

In summary, the road safety is indirectly increased by the enhancement of $\mathrm{V} 2 \mathrm{~V}$ communication link using the proposed system. The remainder of this papers is as follows: the mentioning about problem formulation is presented in Section 2 including optimal half power beamwidth determination. Beam tracking algorithm is also presented in this section. Next, the device configuration and experimental setup are presented in Section 3. The results and discussion are presented in Section 4. Finally, the conclusion is given in Section 5.

\section{Problem Formulation}

2.1. Optimal HPBW Determination. Focusing on optimal HPBW investigation, in this paper, the SQP optimization process has been selected to solve the optimization problem. First of all, the fundamental of proposed system is explained. The output of optimal HPBW investigation is the optimal HPBW values which can provide the maximum RSSI and SNR. As a result, the maximum SNR presents the best quality of $\mathrm{V} 2 \mathrm{~V}$ communication link. According to this, a better communication link refers to a higher RSSI at the receiving side. Thus, the higher RSSI improves system performance and system reliability. This paper proposes 4 -switched-beam system with the optimum HPBW observation. Moreover, the comparison of two different systems is based on the same transmission power for an impartiality comparison between two cases. Thus, the gain comparator factor has been investigated for adjusting the antenna gain function of the two cases in a fairness comparison. The work presented in [28] 

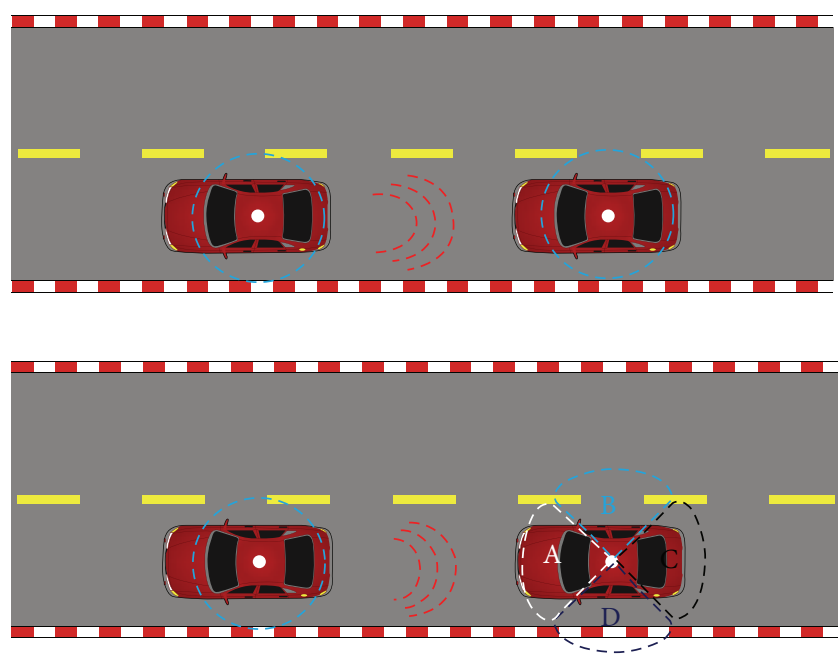

FIGURE 1: Traditional V2V communication when employing omnidirectional antenna.

has shown that the antenna gain function depends on main beam directions. The main beam directions are related to the antenna HPBW. Focusing on the performance metrics of V2V communication system, the work introduced in [29] has presented that the Effective Communication Range (ECR) or $R_{\text {safe }}$ is configured according to BER threshold and PDR threshold related to IEEE802.11p standard. According to this, when vehicle is traveling inside communication area, this vehicle guarantees getting a high link reliability. Moreover, $\operatorname{ECR}\left(R_{\text {safe }}\right)$ can be related to time to collision or $T_{\text {safe }}$ as shown in (1) and (2). The safety range $\left(R_{\text {safe }}\right)$ is related to the speed of the vehicle $(\delta)$ and the PDR value: 0.9 . Note that it can only be used in urban road with asphalt material. In addition, $\left(R_{\text {safe }}\right)$ indicates the distance for the driver preparation to control vehicle into a bad situation on the road. Moreover, the relationship between the proposed system and time to collision or safety time $\left(T_{\text {safe }}\right)$ is presented in (2). According to this, antenna technology is related with $\mathrm{V} 2 \mathrm{~V}$ communication link in terms of Effective Communication Range $\left(R_{\mathrm{ECR}}\right)$. Thus, $\left(R_{\text {safe }}\right)$ and $\left(T_{\text {safe }}\right)$ are important:

$$
\begin{aligned}
R & =\frac{(\delta \cdot 3.6)^{2}}{100}, \\
T_{\text {safe }} & =\frac{R_{\text {safe }}-R}{\delta} .
\end{aligned}
$$

The proposed system includes omnidirectional antenna installing at the transmitting vehicle and the receiving vehicle equipped with switched-beam system following with Figure 1. Also, the relationship between transmitting and receiving signals is presented in

$$
y=h x+n,
$$

where $y$ is a receiving signal, $h$ is a wireless channel, and $x$ is the transmitting signal. From (3), the average received power can be expressed as shown in

$$
P_{r}=|x|^{2} \overline{|h|^{2}}+\overline{|n|^{2}}
$$

where $P_{r}$ is the average received power which combines the multiplication of transmitting signal power and propagation channel coefficient and the average noise power. The channel coefficient is expressed using Friss transmission equation. Then, (4) can be rewritten as shown in (5). Let $N$ be the average noise power as $\overline{|n|^{2}}=N$. As a result, the signal to noise ratio (SNR) can be calculated in (6) and (7). However, there are more buildings and obstacles around the communication zone in an urban road. The scattering signal and interference signal from the nearby radio equipment get impact to the receiving vehicle. According to this, the probabilities of interference effect at the receiving side and the effect from the obstacles increase. This is because the receiving vehicle can receive signals from all directions. Also, the quality of V2V communication link decreased. Thus, V2V communication link in urban area needs the improvement of radio link quality. As a result, high performance link provides a high reliability data exchanging between vehicles. Focusing on the structure of the proposed system, Figure 2 presents the angle parameters $\left(\phi_{r}, \phi_{t}\right)$. Assuming $N\left(\phi_{r}, \phi_{t}\right)=N$; $\forall\left(\phi_{r}, \phi_{t}\right)$ and SNR $=\gamma\left(\phi_{r}, \phi_{t}\right)$, then the average signal to noise ratio depends on the average antenna gain according to vehicle position probability and angle spread between beams as expressed in (6) and (7) as follows:

$$
\begin{aligned}
& P_{r}\left(\phi_{r}, \phi_{t}\right) \\
& \quad=P_{t}\left(\phi_{r}, \phi_{t}\right) G_{t}\left(\phi_{r}, \phi_{t}\right) G_{r}\left(\phi_{r}, \phi_{t}\right)\left(\frac{\lambda}{4 \pi R}\right)^{2} \overline{|h|^{2}}, \\
& \gamma\left(\phi_{r}, \phi_{t}\right)=\frac{P_{r}\left(\phi_{r}, \phi_{t}\right)}{N\left(\phi_{r}, \phi_{t}\right)}, \\
& \gamma\left(\phi_{r}, \phi_{t}\right) \\
& \quad=\frac{P_{t}\left(\phi_{r}, \phi_{t}\right) G_{t}\left(\phi_{r}, \phi_{t}\right) G_{r}\left(\phi_{r}, \phi_{t}\right)(\lambda / 4 \pi R)^{2} \overline{|h|^{2}}}{N} .
\end{aligned}
$$

To find the average value over all directions, the average SNR can be calculated by

$$
\bar{\gamma}=E\left\{\frac{P_{r}\left(\phi_{r}, \phi_{t}\right)}{N}\right\} .
$$

Focusing on the average antenna gain determination, the antenna gain function $\left(G_{i}(\phi)\right)$ consisted of the relationship between HPBW and main beam directions which is expressed in

$$
G_{i}(\phi)=g_{i} \times 10^{0.1\left(G_{\max }-12\left[\left(\phi-\phi_{\mathrm{MB}_{i}}\right) / \mathrm{BW}_{i}\right]^{2}\right)},
$$

where $\left(\phi_{\mathrm{MB}_{i}}-\mathrm{BW}_{i} / 2\right) \leq \phi \leq\left(\phi_{\mathrm{MB}_{i}}+\mathrm{BW}_{i} / 2\right)$. However, another important issue is a comparison of transmission power between three different systems. The comparison is based on equal transmission power. Then, the adjustment factor has been computed by gain comparator parameter $\left(g_{i}\right)$ which will adjust the transmission power equally to an antenna having omnidirectional pattern. Next, the probability density function is expressed in (10) which includes 


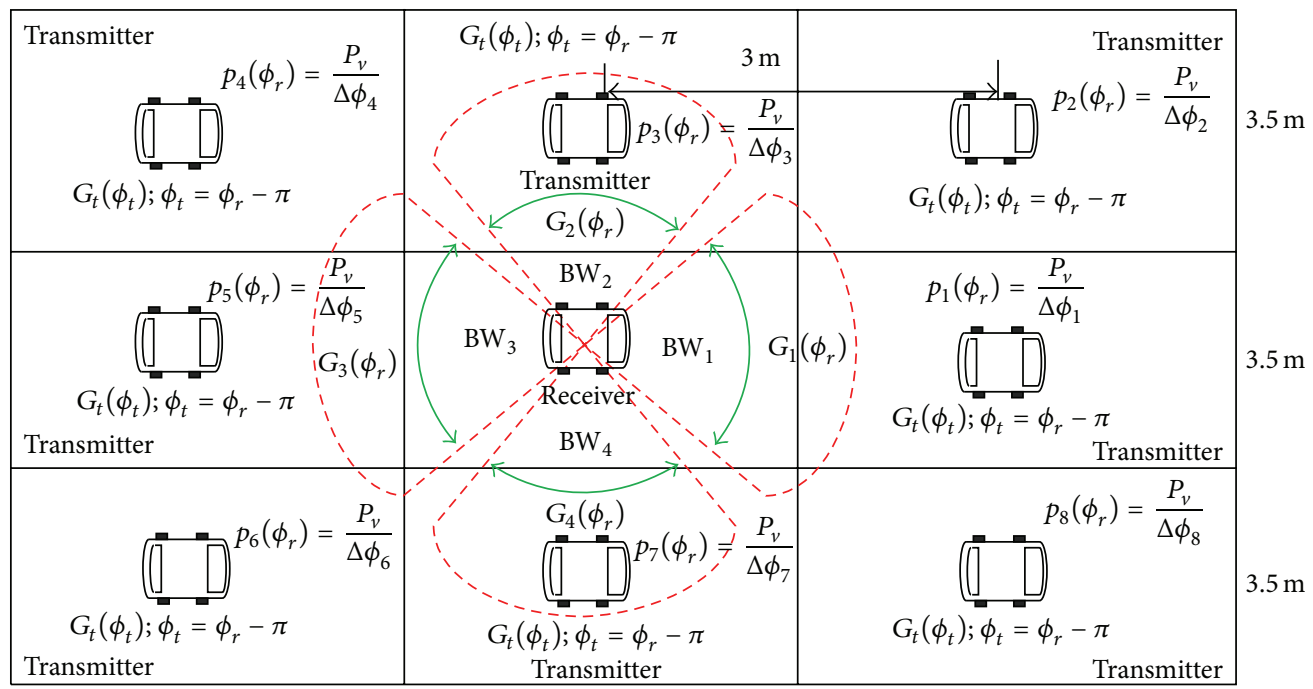

FIGURE 2: Proposed system when applying switched-beam antennas at receiver vehicle.

the vehicle position probability $\left(P_{y}\right)$ and the angle spread between beams $(\Delta \phi)$ :

$$
p_{i}(\phi)=\frac{P_{v}}{\Delta \phi}
$$

where $(\Delta \phi)$ is determined by the road width $\left(W_{r}\right)$ and distance between vehicles $\left(D_{v}\right)$ according to the related works in a previous section. Then, a suitable antenna gain function consisted of HPBW $\left(\mathrm{BW}_{i}\right)$, main beam directions $\left(\mathrm{MB}_{i}\right)$, and probability density function of vehicle position $\left(p_{i}(\phi)\right)$ which are expressed in (10). The angle spread between beams $\Delta \phi$ is expressed in

$$
\Delta \phi=2 \cdot \arctan \frac{\left(W_{r}\right)}{\left(D_{v}\right)} .
$$

These parameters related to the road structure and the distance between vehicles. In addition, the computation of communication link performance is firstly initialized with Friis transmission equation which is expressed in (7). $G_{t}\left(\phi_{r}, \phi_{t}\right)$ and $G_{r}\left(\phi_{r}, \phi_{t}\right)$ are the function of transmitting and receiving antenna gain. The proposed system is considered at the receiving vehicle. Also, $\overline{G_{r}}=(1 / 2 \pi) \int_{0}^{2 \pi} G_{i}(\phi) p_{i}(\phi) \mathrm{d} \phi$ changes to

$$
\overline{G_{r}}=\frac{1}{2 \pi} \int_{0}^{2 \pi} G_{r}\left(\phi_{r}\right) p_{r}\left(\phi_{r}\right) \mathrm{d} \phi_{r} .
$$

The transmitting vehicle is equipped with omnidirectional antenna. V2V safety message disseminates to the receiving vehicle. The receiving vehicle is installed with switched-beam antenna system. The distance between two vehicles is presented in terms of $R$. The related parameters are described as the following. Let $P_{t}\left(\phi_{r}, \phi_{t}\right)=P_{t}, G_{t}\left(\phi_{r}, \phi_{t}\right)=$ $G_{t}\left(\phi_{t}\right)$, and $G_{r}\left(\phi_{r}, \phi_{t}\right)=G_{r}\left(\phi_{r}\right)$. When the relationship between $\phi_{r}$ and $\phi_{t}$ is $\phi_{t}=\phi_{r}-\pi$. Then, signal to noise ratio can be calculated using (8). The relationship between $\phi_{r}$ and $\phi_{t}$ is $\phi_{t}=\phi_{r}-\pi$. As a result, (5) is changed to (13) as follows:

$$
\begin{aligned}
& P_{r}\left(\phi_{r}, \phi_{t}\right)=P_{r}\left(\phi_{r}\right)=P_{t} G_{t}\left(\phi_{t}\right) \overline{G_{r}\left(\phi_{r}\right)}\left(\frac{\lambda}{4 \pi R}\right)^{2} \overline{|h|^{2}}, \\
& P_{r}\left(\phi_{r}\right)=P_{t} G_{t}\left(\phi_{r}-\pi\right) \overline{G_{r}\left(\phi_{r}\right)}\left(\frac{\lambda}{4 \pi R}\right)^{2} \overline{|h|^{2}}, \\
& \bar{\gamma}=E\left\{\frac{P_{t} G_{t}\left(\phi_{r}-\pi\right) \overline{G_{r}\left(\phi_{r}\right)}(\lambda / 4 \pi R)^{2}}{N}\right\} \overline{|h|^{2}}, \\
& \bar{\gamma}=\left(\frac{P_{t}}{N}\right) \overline{|h|^{2}}\left(\frac{\lambda}{4 \pi R}\right)^{2} \\
& \quad \cdot \int_{0}^{2 \pi} G_{t}\left(\phi_{r}-\pi\right) \overline{G_{r}\left(\phi_{r}\right)} p\left(\phi_{r}\right) \mathrm{d} \phi_{r}, \\
& \bar{\gamma}=\left(\frac{P_{t}}{N}\right) \overline{|h|^{2}}\left(\frac{\lambda}{4 \pi R}\right)^{2} \int_{0}^{2 \pi} G_{r}\left(\phi_{r}\right) p\left(\phi_{r}\right) \mathrm{d} \phi_{r}, \\
& \operatorname{BER}_{\mathrm{BPSK}}=\frac{1}{2} \operatorname{erfc}\left(\sqrt{\frac{\bar{\gamma}}{2}}\right), \\
& \operatorname{PER}^{2} 1-(1-\mathrm{BER})^{8 \times L} .
\end{aligned}
$$

The noise power level $N$ is gathered from the real measurements in urban road area. These values are collected from 5 times per point. The performance metrics indicate the Effective Communication Range $\left(R_{\text {safe }}\right)$. The vehicles traveling inside a communication area maintains a stable link according to BER and PER threshold. Focusing on IEEE802.11p standard, PDR is configured at $90 \%$. This value introduces more link reliability. The expression in (18) indicates the result of HPBW for V2V communication link. Also, the expression in (19) introduces the relationship between PER and BER, 
where $L$ refers to the packet size. The work presented in [30] has introduced that the relationship between BER and PER relates with packet length. Moreover, PER causes a wrong decision of the driver when receiving high PER of safety message. Also, PER and BER are the performance metrics which indicate the performance of vehicular network.

The optimum HPBW determination process initiates with the constraint function which checks the condition of input values. Also, the average antenna gain and average SNR are calculated inside the objective function. The proposed systems can be categorized into nonlinear multivariable optimization. The works presented in $[31,32]$ have introduced Jacobian and Lagrange methods which are suitable for a single equality constraint. Focusing on the structure of the optimization problem, the proposed system requires a correction of antenna gain function corresponding to the vehicle position on the road. The selection of suitable antenna gain function, vehicle position probability, and the angle spread between beams introduce that the mentioning methods from above are improper for the proposed system. Moreover, the difficulty of the computation presents the unsuitableness for the proposed optimization problem. The work presented in [33] has expressed that the proper method for the similar objective function as the proposed system is SQP method. SQP method is the powerful method for the determination of the optimum value for the objective function which is related with quadratic equation. Moreover, SQP method requires low processing time. Also, SQP optimization method is selected for optimal HPBW determination. To apply SQP method for the determination of optimum HPBW for V2V communication, the objective function can be given in (20). This function consisted of input HPBW values, exact antenna gain function, and probability density function of vehicle position probability:

$$
\begin{aligned}
f & \left(\mathrm{BW}_{1}, \ldots, \mathrm{BW}_{M}\right) \\
& =\left(\frac{P_{t}}{N}\right) \overline{|h|^{2}}\left(\frac{\lambda}{4 \pi R}\right)^{2} \int_{0}^{2 \pi} G_{r}\left(\phi_{r}\right) p\left(\phi_{r}\right) \mathrm{d} \phi_{r},
\end{aligned}
$$

where $M$ is the number of beams. The solution can be obtained by applying SQP method on the optimization problem as presented in

$$
\begin{array}{ll}
\text { Maximize: } & f\left(\mathrm{BW}_{1}, \ldots, \mathrm{BW}_{M}\right) \\
\text { Subject to: } & \sum_{i=1}^{M} \mathrm{BW}_{i}=2 \pi .
\end{array}
$$

A determination of the maximum average SNR is the main purpose of the objective function according to the constraint which is shown in (22). The summation of input HPBW values is equal to 360 degrees. The simulation program computes all cases according to the optimization constraint to guarantee the goal of objective function. Also, the maximum average SNR indicates the best solution of HPBW values. Then, the best solution of HPBW producing a maximum average SNR is selected to be the optimum HPBW. Moreover, the radiation pattern of the optimum

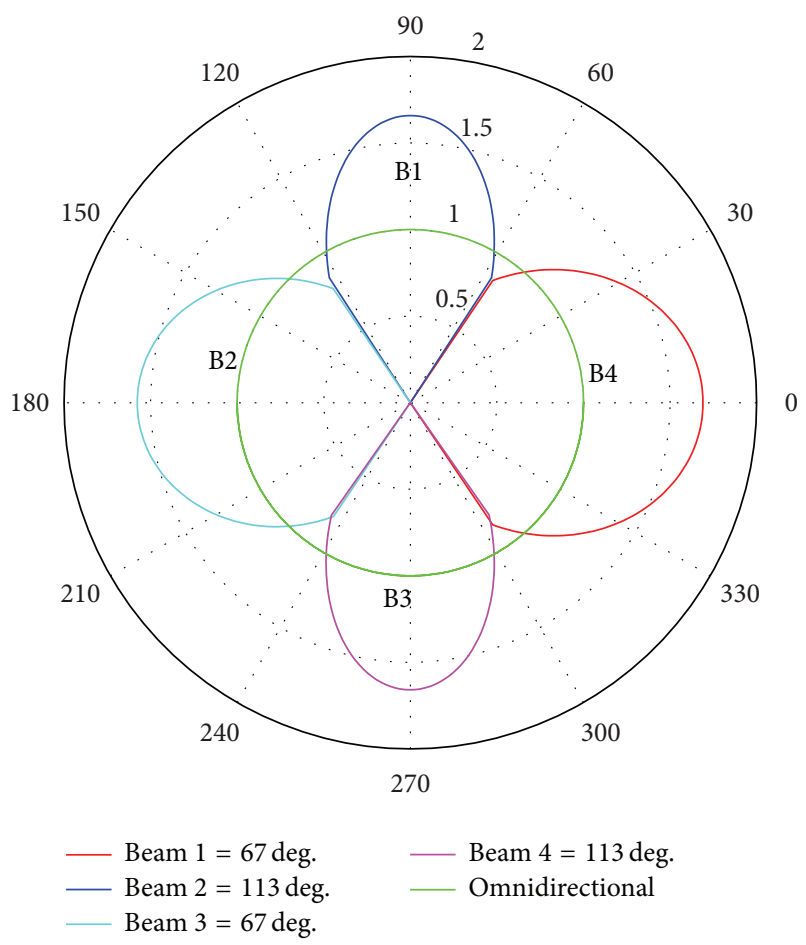

FIGURE 3: Radiation pattern of the proposed system (simulation).

HPBW is shown by Figure 3 including with 67, 113, 67, and 113 degrees of Beam 1, Beam 2, Beam 3, and Beam 4, respectively. Then, $\mathrm{V} 2 \mathrm{~V}$ communication link reliability can be computed. Moreover, the performance evaluations in terms of ECR $\left(R_{\text {safe }}\right)$ and TTC $\left(T_{\text {safe }}\right)$ corresponding to IEEE802.11p standard can be calculated via (19). Moreover, this paper introduces the measured radiation pattern of the optimal HPBW presented in Figure 4. The experiments introduced the improving of $\mathrm{V} 2 \mathrm{~V}$ communication link performance in terms of RSSI. However, the information of beam direction is another significant parameter to ensure that the receiving vehicle can receive DSRC message according to the proper beam. Also, beam tracking algorithm will be explained in next subsection.

2.2. Beam Tracking Algorithm. The determination of optimal HPBW has been presented in the previous section. Also, another important issue for $\mathrm{V} 2 \mathrm{~V}$ communication link is beam tracking. This section introduced beam tracking mechanism using RSSI decision-based algorithm. The work presented in $[20-23,34]$ has introduced that the signal strength of the receiving signal depends on path loss, shadowing fading, and multipath fading. Focusing on path loss issue, path loss is directly proper with the distance between vehicles. The increasing of distance between vehicles reduces the signal strength at the receiving vehicle. Focusing on urban road environments, many obstacles locate near the communication zone. Also, the effect from obstacles occurs at the receiving side. Many researchers try to improve the signal street at the receiving side under the mentioned conditions. Also, the abovementioned indicate the significance of improving 


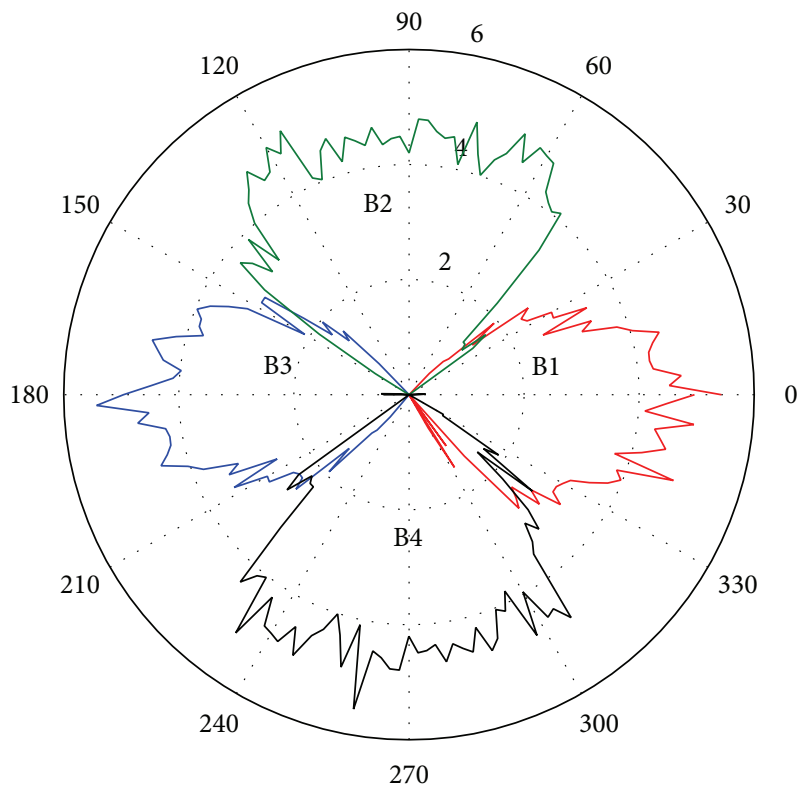

(a)

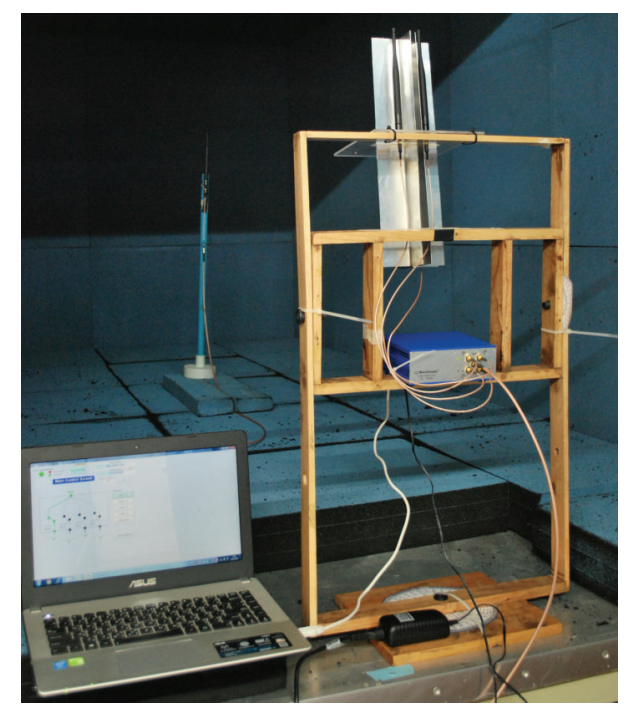

(b)

FIgURE 4: (a) Measured radiation pattern. (b) Devices and radiation pattern measurement.

V2V communication link. The previous section presented the powerful method of finding the optimum HPBW producing a good signal quality at the receiving side. However, to ensure that $\mathrm{V} 2 \mathrm{~V}$ communication link will connect all the time and that there is no connection loss in $\mathrm{V} 2 \mathrm{~V}$ communication link, beam tracking algorithm for $\mathrm{V} 2 \mathrm{~V}$ is important. The study cases can be separated into main scenarios: (1) Beam 1 changes to Beam 2 and (2) Beam 2 changes to Beam 3. The decision mechanism is based on (24).

For V2V communication using Beam 2, Beam 1 changes to Beam 2:

$$
\begin{aligned}
& P_{\text {rxb1 }}<P_{\mathrm{Th}}, \\
& P_{\text {rxb2 }}>P_{\text {rxmin }} .
\end{aligned}
$$

For V2V communication using Beam 3, Beam 2 changes to Beam 3:

$$
\begin{aligned}
& P_{\text {rxb2 }}<P_{\mathrm{Th}}, \\
& P_{\text {rxb3 }}>P_{\text {rxmin }} .
\end{aligned}
$$

Beam 4 has been neglected due to the road structure. Only two-lane road is interesting in this paper. Since these events are statistically independent, $P_{\mathrm{B} 1}, P_{\mathrm{B} 2}$, and $P_{\mathrm{B} 3}$ are presented as the following:

$$
\begin{aligned}
& P_{\mathrm{B} 1}=Q\left(\frac{\mu_{1}-P_{\mathrm{Th}}}{\sigma}\right) Q\left(\frac{P_{\mathrm{rxmin}}-\mu_{2}}{\sigma}\right), \\
& P_{\mathrm{B} 2}=Q\left(\frac{\mu_{2}-P_{\mathrm{Th}}}{\sigma}\right) Q\left(\frac{P_{\mathrm{rxmin}}-\mu_{1}}{\sigma}\right), \\
& P_{\mathrm{B} 3}=Q\left(\frac{\mu_{3}-P_{\mathrm{Th}}}{\sigma}\right) Q\left(\frac{P_{\mathrm{rxmin}}-\mu_{2}}{\sigma}\right),
\end{aligned}
$$

where $P_{\mathrm{Th}}=-88 \mathrm{dBm}$ and $P_{\text {rxmin }}=-95 \mathrm{dBm}$ refer to receiving power threshold and minimum receiving power at the receiving vehicle. In addition, $\mu_{1}=K_{1}-K_{2}$, $\mu_{2}=K_{1}-K_{2} \times \log _{10}\left(R-d_{1}\right)$, and $\mu_{3}=K_{1}-K_{2} \times$ $\log _{10}\left(R-d_{2}\right)$ are mean of the receiving power presenting in Gaussian process. $d_{1}, d_{2}$ are the distance between vehicles. $R$ is DSRC communication range which equals $1000 \mathrm{~m}$. Moreover, $\sigma$ introduces the standard deviation of shadowing effect according to IEEE802.11p (WAVE-DSRC) standard. $\sigma$ has been varied for the simulation of beam selection probability. Also, $K_{1}$ and $K_{2}$ are path losses factor. The work in [20] has presented that $K_{1}$ and $K_{2}$ are equal to $0 \mathrm{~dB}$ and $30 \mathrm{~dB}$, respectively. The probability of beam selection is presented in Figures 5 and 6. Due to the connection loss issue, also the "Make before Break" algorithm has been selected to ensure that there is no connection loss in $\mathrm{V} 2 \mathrm{~V}$ communication link. The simulation results in Figures 5 and 6 present the beam selection probability according to the proposed algorithm.

\section{Devices Configuration and Experimental Setup}

The experimental equipment is as the following: two vehicles with the same types, two Locomate OBU which are equipped with omnidirectional antenna. One Locomate device is installed at the transmitting vehicle which is configured as the Provider. Another one for receiving vehicle which is setup as User. These devices are installed at the center of the vehicle's roof. The transmitting vehicle installs the antenna having omnidirectional pattern. Focusing on the receiving vehicle, the proposed system is installed with corner reflector for producing HPBW according to optimal HPBW value. The main beam of each antenna steers to $0-, 90-, 180-$, and 360degree directions. The SWB system steers the beam every 


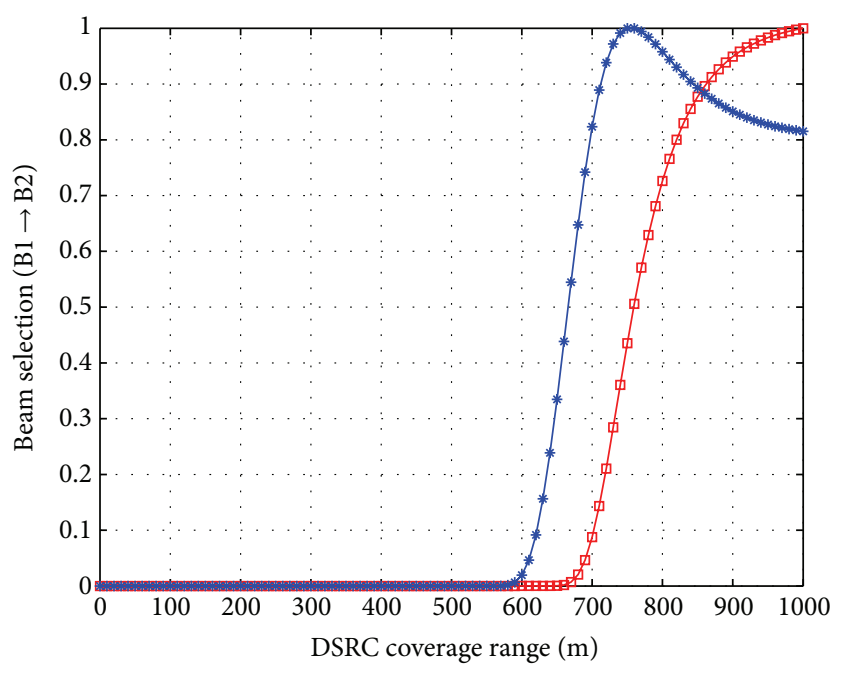

$\square-$ Beam 1

$\rightarrow$ - Beam 2

FIGURE 5: Beam selection probability Beam 1-Beam 2.

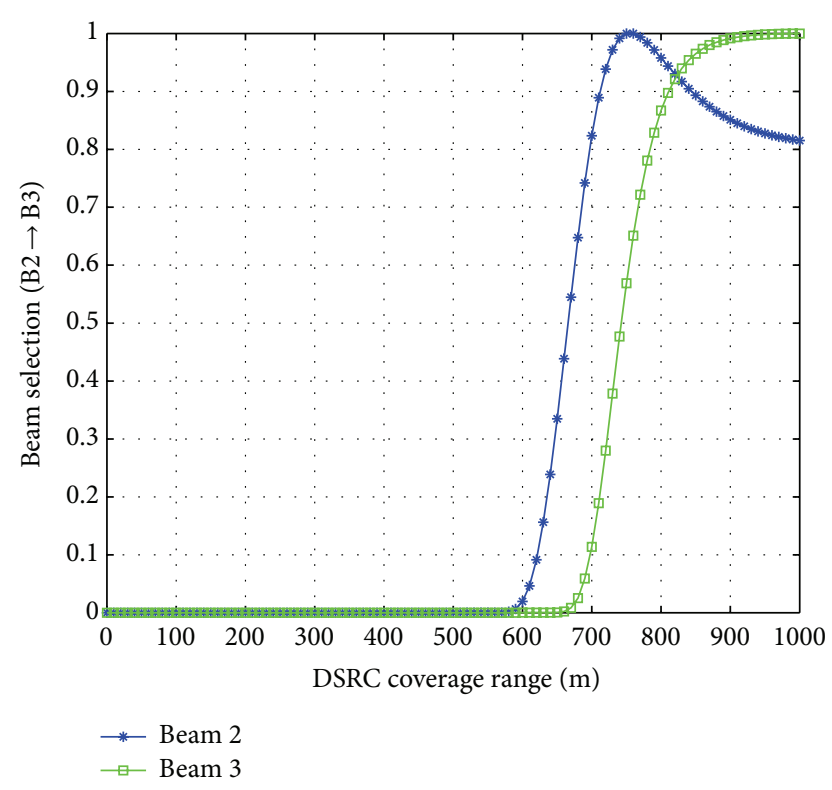

FIgURE 6: Beam selection probability Beam 2-Beam 3.

$100 \mathrm{~ms}$ from Beam 1 to Beam 4. This is because the switches time relates to the channel switching time of DSRC standard. As a result, signal from antenna with corner reflector is fed into OBU. Also, OBU connect with host computer which holds the maximum RSSI. As a result, the receiving vehicle can also receive a good signal quality due to the manner of SWB mechanism. However, when the vehicle is moving out of the communication rage, the communication link between two vehicles is discarded. Finally, the SWB system starts for a new process but using the same procedure. The comparing between conventional V2V communication link performance and SWB system is the major purpose for next experiments. The road is made of asphalt which is related to the previous section that mentioned safety distance in an urban road. The experimental setup is configured as two vehicles travelling in the same lane. The vehicle in the front is configured as the receiving vehicle which is equipped with SWB system. The vehicle in the back is configured as the transmitting vehicle. The length of message size is configured as 512 bytes. The transmitting vehicle travels following the vehicle position (Point numbers 1 to 11 ) due to the test of beam tracking. RSSI of the reeving signal have been collected 5 times per position. The results of experiments are presented in the next section.

\section{Experimental Results and Discussion}

In Figure 7, the results present the relationship between vehicles positions and beam indices. Focusing on positions $1-4$, Beam 1 has been selected for V2V data transmission. Moreover, the connected beam changed when the receiving power is relating to (23) and (24). Beam 1 changes to Beam 2 when the vehicle traveling passed positions 5-7. To choose the right beam index, the vehicle position should be accurately estimated. In our experiment, the estimation error is very little which does not affect the beam selection. However, vehicle position estimation error impacts on the performance metric when changing beam index. Vehicle position estimation error introduces incorrect beam selection. As a result, RSSI at the receiving vehicle reduces. Then, the communication between vehicles is reconnected. Also, new process of beam tracking is initiated. Then, the delay of the mentioned process occurs. The increasing of delay increases the latency of $\mathrm{V} 2 \mathrm{~V}$ data dissemination. After that, Beam 2 changes to Beam 3 when the vehicles traveling passed positions $8-11$. The performance of receiving signal has been improved due to the information of beam tracking. Moreover, the proper connected beam introduces increasing of RSSI at the receiving side. In addition, the beam tracking provides a low connection loss to maintain a continuous V2V communication link. As a result, the reliability of information exchange between vehicle is increased. The result also shows that beam tracking provides a correct connected beam which provides a good signal quality at the receiving vehicle.

Figure 8 presents RSSI performance comparing between conventional system and the proposed system. Among antenna cases in the results, the optimum HPBW case indicates the best performance in terms of RSSI, $R_{\text {Safe }}$, and $T_{\text {Safe }}$ performances. This is because the HPBW of the antenna is optimally allocated to the position of the vehicles on the road. Focusing on $600 \mathrm{~m}$ distance between vehicles, the proposed system increases RSSI performance $5 \mathrm{~dB}$ or three times from the conventional system. The result indicates the increasing of coverage range which is related to the cell planning for IEEE802.11p in [35]. The improvement of RSSI is benefit for coverage optimization planning which is significant for the deployment of WAVE-DSRC network.

$R_{\text {Safe }}$ performance is presented in Figure 9. As a result, the optimum HPBW case provides more $R_{\text {Safe }}$ in comparing to the conventional case. Moreover, the proposed case is better than conventional case for $35.5 \%$. This result indicates that the vehicles using switched-beam with optimum HPBW system and beam tracking algorithm can receive the safety message 

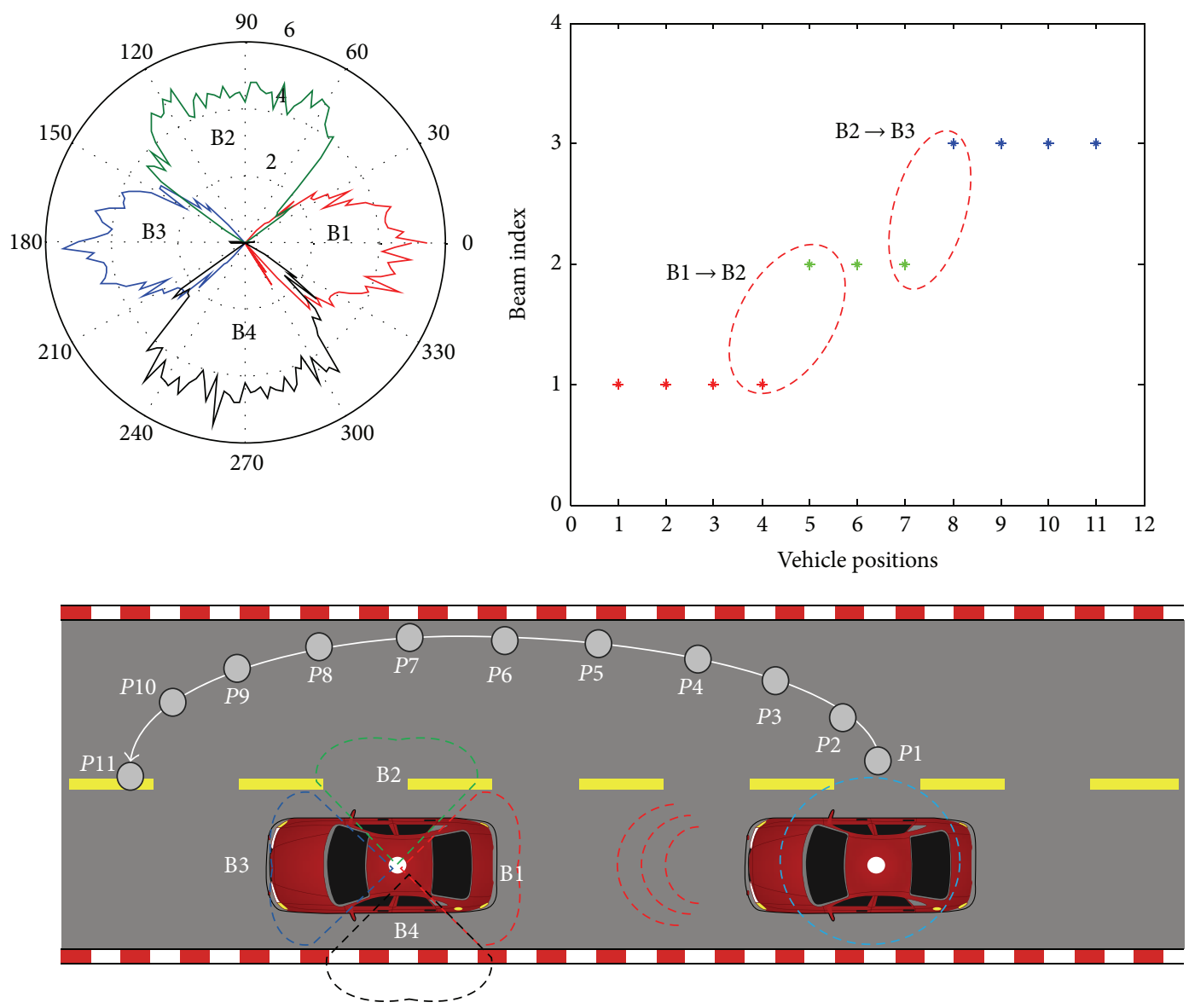

FIGURE 7: The comparison of beam index and vehicle positions on the road for beam tracking algorithm.

in a longer range when comparing to the conventional case. Also, the drivers inside the receiving vehicle can have a better chance to control a car in a bad situation. As a result, the proposed system increases the traffic safety by using switched-beam with optimum HPBW and beam tracking. Focusing on the vehicle speed at $60 \mathrm{~km} / \mathrm{hr}$, the optimum HPBW offers the best performance when comparing to the conventional system. Another performance metrics in terms of time to collision is presented by Figure 10. $T_{\text {safe }}$ of the optimum HPBW is better than conventional case. This result confirms the effective usage of switched-beam system which can enhance the time to collision performance. Focusing on the real situation, the driver drives a vehicle at $60 \mathrm{~km} / \mathrm{hr}$ and moves to the dangerous situation on the road. Preparing time for a bad situation increases 5 seconds when using proposed beamforming system. The result indicates the road safety increasing by the proposed beamforming and beam tracking approaches. Then V2V communication system with optimum HPBW disseminates the warning messages to the driver for a long communication range. As a result, the driver has more times for braking or preparing to the dangerous situation on the road. Also, the traffic safety has been increased when applying switched-beam antenna with the optimum HPBW and beam tracking to V2V application.

Another performance metric is packet error rate (PER) as presented in Figure 11. The result indicates that PER of the proposed system is less than $25 \%$ comparing to the conventional system at $300 \mathrm{~m}$. Assume that 100 packets' transmission and 40 packets' error occur at the receiving side when using conventional system. But 15 packets' error occurs at the proposed system. The results indicate an indirect impact of the communication reliability. Focusing on safety message dissemination, the proposed system receives for more correct packet comparing to the conventional system. Also, safety message transmission with high reliability introduces the increasing of warning message accuracy. Thus, road safety increases due to the benefit of the proposed system. The comparative study in terms of PER performance metric indicates the successful beamforming and tracking approaches. Moreover, Bit Error Rate (BER) is the significant parameters of the communication link. The result shown in Figure 12 indicates the comparison of BER and distance between vehicles. The authors employ the approximation of BER introduced in [30]. Receiving powers of the proposed system are measured by the experiment on urban road environment. Two-lane road has been chosen. OBU with beamforming system is installed at the receiving vehicle. Another OBU with omnidirectional antenna is configured as transmitting vehicle. The receiving power was measured 5 times per points. Also, the average receiving power has been calculated with average noise power. Then, SNR has been translated to Eb/N0. Focusing at $300 \mathrm{~m}$ of distance between 


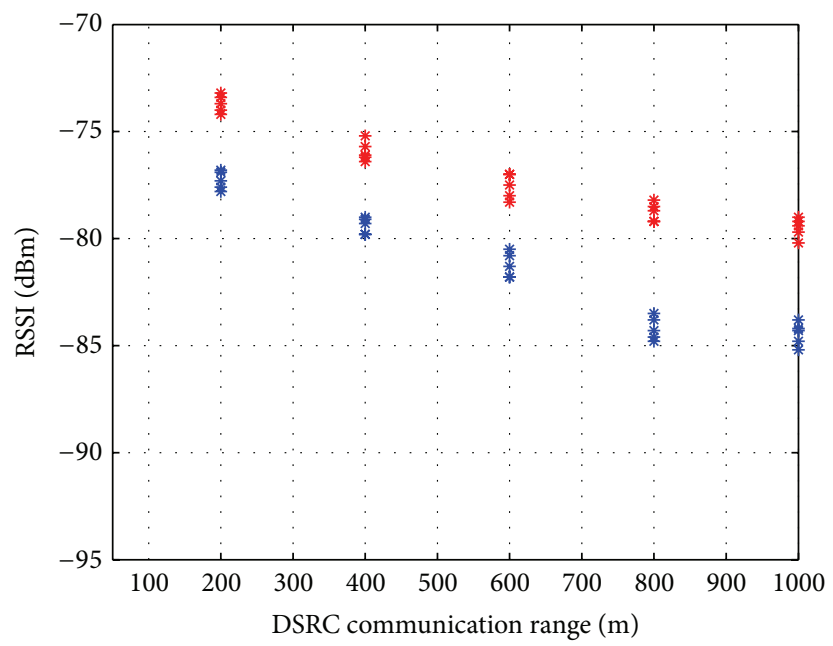

(a)
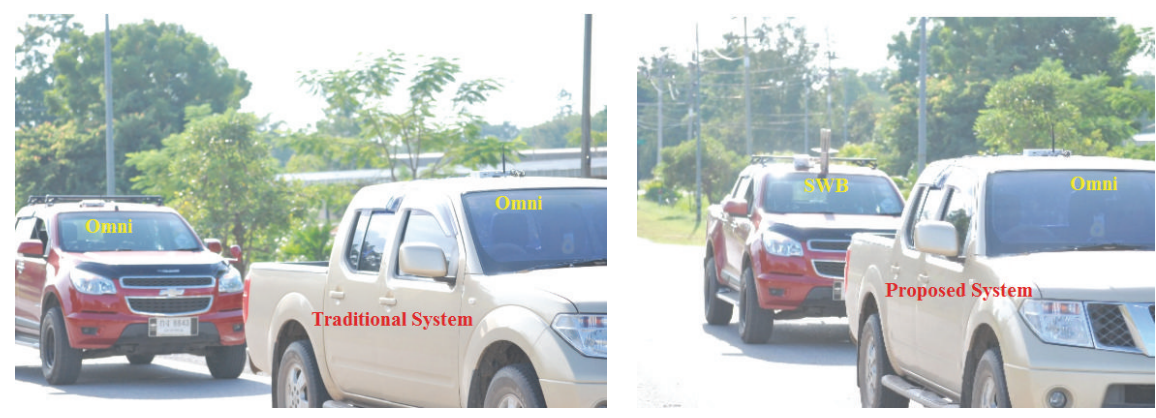

(b)

FIGURE 8: (a) RSSI performance when comparing between conventional system and SWB system. (b) The real experiment on the road.

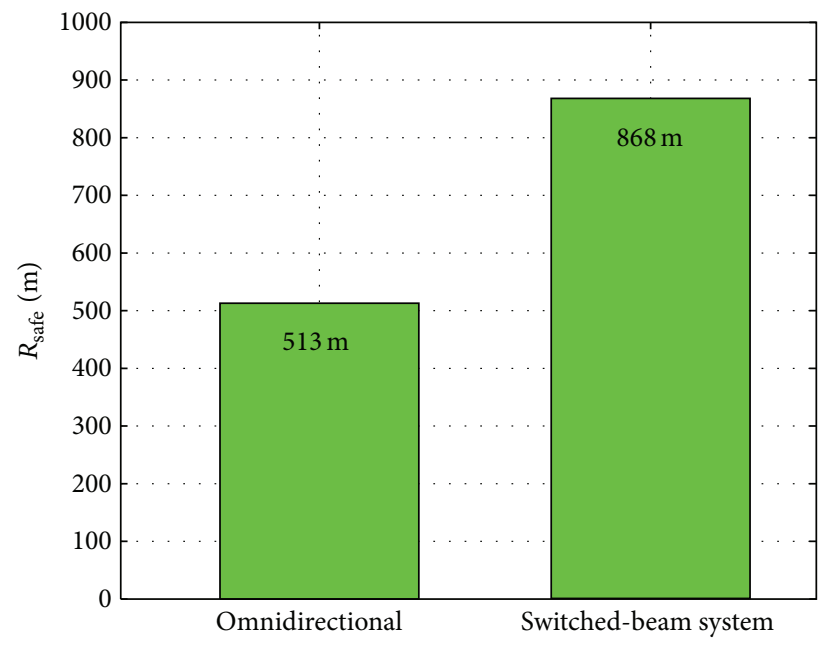

FIgure 9: Effective Communication Range for DSRC V2V communication.

vehicles and 100 bytes of transmission packet size, BER of the conventional system is $4 \times 10^{-2}$. BER of the proposed system is $2 \times 10^{-4}$. As a result, the amount of bit error for the conventional system is 32 bits more than the proposed beamforming system. High BER indicates unreliable link. As a result, road safety is reduced due to the impact of incorrect warning message reception. Focusing on another QOS metrics, throughput can be estimated via the approximation method. The configuration is as follows: bandwidth: $10 \mathrm{MHz}$, data rate: $27 \mathrm{Mbps}$, modulation type: OFDM (BPSK), channel number: 172, frequency: $5.9 \mathrm{GHz}$, and devices: Locomate OBU devices. The result in Figure 13 indicates the improving of system throughput by using beamforming and tracking approaches. The throughput of proposed system provides $1 \mathrm{Mbps}$ more than the conventional system. This means that the proposed system can transmit message faster than the conventional system. Focusing on the real life scenario, a huge message size such as short period video and high-quality photo from car DVR (car Digital Video Recording) can transmit with higher speed than conventional system. The processing unit of those video and photo files can process and transmit the warning message faster than the conventional system. The impact of the proposed system increases the road safety indirectly.

\section{Conclusion and Future Work}

This paper presents the importance of applying optimum HPBW for V2V DSRC application. Switched-beam system with optimum HPBW enhances the communication link 


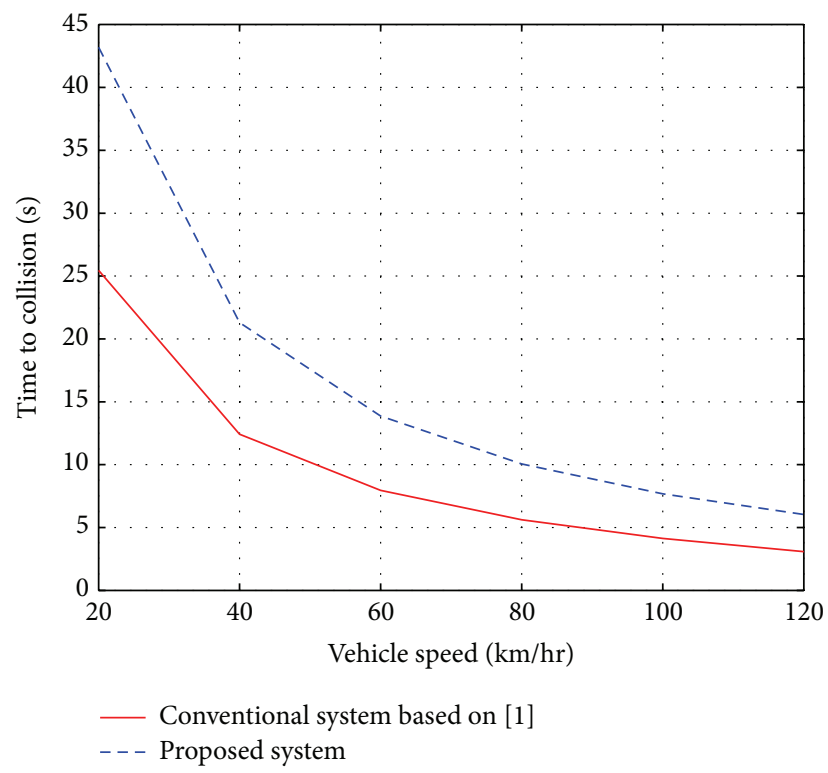

FIGURE 10: Time to collision performance when comparing between conventional system and SWB system.

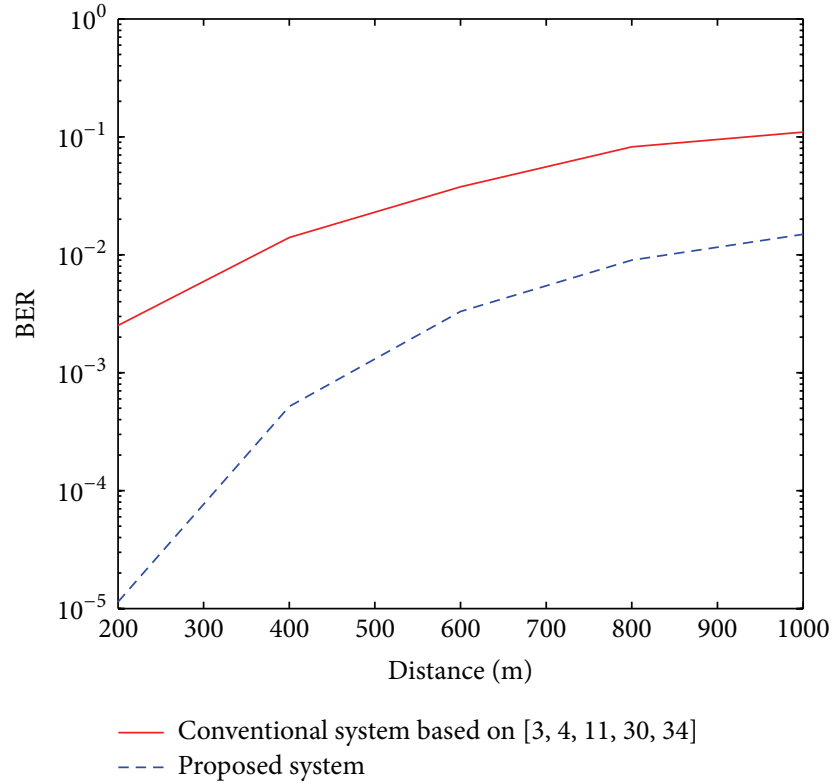

FIGURE 11: Packet error rate comparing to distance between vehicles.

between vehicles. The optimization problem is mathematically modeled by considering the probability of vehicle positions as well as road environments. SQP optimization method has been proposed for optimum HPBW determination. Moreover, beam tracking algorithm has been applied to ensure a seamless connection between vehicles. The results indicate that applying switched-beam antenna with optimum HPBW and beam tracking mechanism for V2V DSRC communication can increase the signal quality and system performances at the receiving vehicle in terms of RSSI, $R_{\text {Safe }}$, and $T_{\text {safe }}$. Particularly, the proposed system can improve

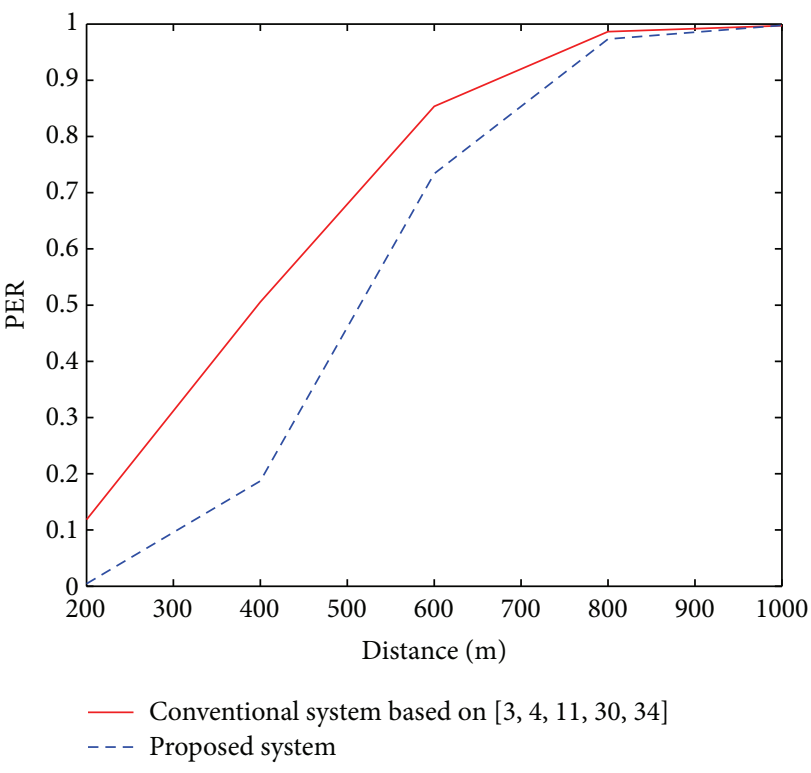

FIGURE 12: Bit Error Rate comparing to distance between vehicles.

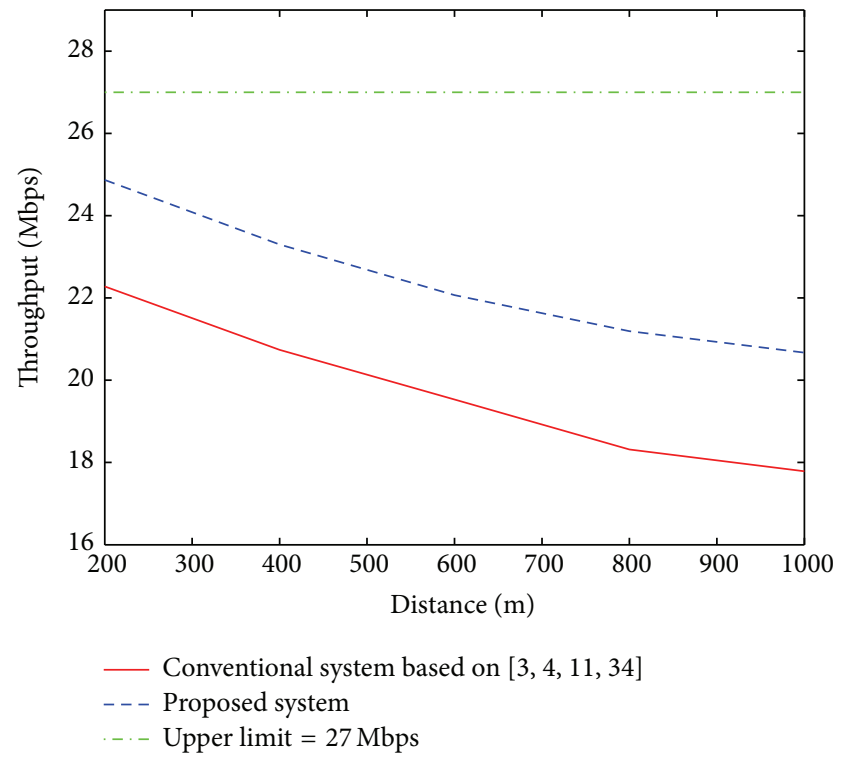

FIGURE 13: Throughput comparing to distance between vehicles.

the traffic safety $74 \%$ in comparing to the conventional DSRC $\mathrm{V} 2 \mathrm{~V}$ communication. This paper has addressed the simple method to save more lives by adjusting the optimum HPBW. Also, the proposed system can be practically implemented on existing V2V technology.

\section{Conflict of Interests}

The authors declare that there is no conflict of interests regarding the publication of this paper. 


\section{Acknowledgment}

This work is financially supported by the RGJ: Royal Golden Jubilee Scholarship, TRF Fund (Grant no. PHD/0041/2553).

\section{References}

[1] L. Zhao, X. Hong, J. Zhang, Y. Zhang, and Q. Hao, "Feasibility analysis of multi-radio in DSRC vehicular networks," in Proceedings of the 16th International Symposium on Wireless Personal Multimedia Communications (WPMC '13), pp. 1-6, IEEE, Atlantic City, NJ, USA, June 2013.

[2] M.-W. Li, T.-H. Wu, W.-Y. Lin, K.-C. Lan, C.-M. Chou, and C.H. Hsu, "On the feasibility of using 802.11p for communication of electronic toll collection systems," in Proceedings of the International Conference on Network-Based Information Systems (NBiS '11), pp. 68-75, Tirana, Albania, September 2011.

[3] J. B. Kenney, "Dedicated short-range communications (DSRC) standards in the United States," Proceedings of the IEEE, vol. 99, no. 7, pp. 1162-1182, 2011.

[4] Y. L. Morgan, "Notes on DSRC \& WAVE standards suite: its architecture, design, and characteristics," IEEE Communications Surveys \& Tutorials, vol. 12, no. 4, pp. 504-518, 2010.

[5] C. R. Dow, M. H. Ho, Y. H. Lee, and S. F. Hwang, "Design and implementation of a DSRC based vehicular warning and notification system," in Proceedings of the IEEE 13th International Conference on High Performance Computing and Communications (HPCC '11), pp. 960-965, Alberta, Canada, September 2011.

[6] Q. Xu, T. Mak, J. Ko, and R. Sengupta, "Vehicle-to-vehicle safety messaging in DSRC," in Proceedings of the 1st ACM International Workshop on Vehicular Ad Hoc Networks, pp. 19-28, ACM, October 2004.

[7] V. Ratanavaraha and S. Suangka, "Impacts of accident severity factors and loss values of crashes on expressways in Thailand," IATSS Research, vol. 37, no. 2, pp. 130-136, 2014.

[8] V. Ratanavaraha and S. Jomnonkwao, "Community participation and behavioral changes of helmet use in Thailand," Transport Policy, vol. 25, pp. 111-118, 2013.

[9] R. Sabouni and R. M. Hafez, "Performance of DSRC for $\mathrm{V} 2 \mathrm{~V}$ communications in urban and highway environments," in Proceedings of the 25th IEEE Canadian Conference on Electrical \& Computer Engineering (CCECE '12), pp. 1-5, IEEE, Montreal, Canada, May 2012.

[10] F. Bian, A. Goel, C. S. Raghavendra, and X. Li, "Energy-efficient broadcasting in wireless ad hoc networks lower bounds and algorithms," Journal of Interconnection Networks, vol. 3, no. 03n04, pp. 149-166, 2002.

[11] R.-T. Juang, "Performance analysis of V2V DSRC communications with reconfigurable antenna," in Proceedings of the 19th ITS World Congress, Vienna, Austria, October 2012.

[12] N.-C. Wang and Y.-C. Huang, "An SDMA-based MAC protocol for wireless ad hoc networks with smart antennas," Computers \& Electrical Engineering, vol. 41, pp. 383-394, 2015.

[13] A. P. Subramanian, V. Navda, P. Deshpande, and S. R. Das, "A measurement study of inter-vehicular communication using steerable beam directional antenna," in Proceedings of the 5th ACM International Workshop on VehiculAr Inter-NETworking (VANET '08), pp. 7-16, San Francisco, Calif, USA, September 2008.

[14] S. Panngam, P. Uthansakul, and M. Uthansakul, "Performance of switched-beam antennas for wireless mesh networks using synchronous collision resolution protocol," in Proceedings of the International Conference on Computer and Information Application (ICCIA '10), pp. 430-433, IEEE, Tianjin, China, November 2010.

[15] M. Uthansakul, P. Chaipanya, and P. Uthansakul, "Performance evaluation of a low-cost switched-beam antenna for WLAN users," Microwave and Optical Technology Letters, vol. 52, no. 9, pp. 2069-2074, 2010.

[16] S. A. Mitilineos and C. N. Capsalis, "A new, low-cost, switched beam and fully adaptive antenna array for $2.4 \mathrm{GHz}$ ISM applications," IEEE Transactions on Antennas and Propagation, vol. 55, no. 9, pp. 2502-2508, 2007.

[17] S. K. Sanyal, Q. M. Alfred, and T. Chakravarty, "A novel beamswitching algorithm for programmable phased array antenna," Progress in Electromagnetics Research, vol. 60, pp. 187-196, 2006.

[18] Z. Zhang, F. Liu, W. Chen, Z. Feng, and W. Xiang, "An endfire phased array used in Wireless Access for Vehicular Environments (WAVE)," in Proceedings of the International Conference on Microwave and Millimeter Wave Technology (ICMMT '08), vol. 1, pp. 428-431, Nanjing, China, April 2008.

[19] F. Liu, Z. Zhang, W. Chen, Z. Feng, and M. F. Iskander, "An endfire beam-switchable antenna array used in vehicular environment," IEEE Antennas and Wireless Propagation Letters, vol. 9, pp. 195-198, 2010.

[20] S. D. Roy, "Performance evaluation of signal strength based handover algorithms," International Journal of Communications, Network and System Sciences, vol. 2, no. 7, pp. 657-663, 2009.

[21] S. Hamdoun, A. Rachedi, and A. Benslimane, "RSSI-based localization algorithms using spatial diversity in wireless sensor networks," International Journal of Ad Hoc and Ubiquitous Computing, vol. 19, no. 3-4, pp. 157-167, 2015.

[22] K.-I. Itoh, S. Watanabe, J.-S. Shih, and T. Sato, "Performance of handoff algorithm based on distance and RSSI measurements," IEEE Transactions on Vehicular Technology, vol. 51, no. 6, pp. 1460-1468, 2002.

[23] N. Zhang and J. M. Holtzman, "Analysis of handoff algorithms using both absolute and relative measurements," IEEE Transactions on Vehicular Technology, vol. 45, no. 1, pp. 174-179, 1996.

[24] C. Balanis, Antenna Theory: Analysis and Design, John Wiley \& Sons, 2012.

[25] F. Bai and K. Hariharan, "Reliability analysis of DSRC wireless communication for vehicle safety applications," in Proceedings of the IEEE Intelligent Transportation Systems Conference (ITSC '06), pp. 355-362, Toronto, Canada, September 2006.

[26] K. A. Hafeez, L. Zhao, B. Ma, and J. W. Mark, "Performance analysis and enhancement of the DSRC for VANET's safety applications," IEEE Transactions on Vehicular Technology, vol. 62, no. 7, pp. 3069-3083, 2013.

[27] Speed limits by country, 2012, https://en.wikipedia.org/wiki/ Speed_limits_by_country.

[28] O. Klemp, "Performance considerations for automotive antenna equipment in vehicle-to-vehicle communications," in Proceedings of the 20th URSI International Symposium on Electromagnetic Theory (EMTS '10), pp. 934-937, Berlin, Germany, August 2010.

[29] K. Xu, B. T. Garrison, and K.-C. Wang, "Performance modeling for IEEE 802.11 vehicle-to-infrastructure networks with directional antennas," in Proceedings of the IEEE Vehicular Networking Conference (VNC '10), pp. 215-222, IEEE, Jersey City, NJ, USA, December 2010. 
[30] H. Alturkostani, Ch. Anup, R. Robert, and K. Axel, "On the design of jamming-aware safety applications in VANETs," in Proceedings of the ACM 10th Annual Cyber and Information Security Research Conference (CISR '15), Oak Ridge, Tenn, USA, April 2015.

[31] D. E. Kirk, Optimal Control Theory: An Introduction, Courier Corporation, 2012.

[32] H. P. Geering, Optimal Control with Engineering Applications, vol. 113, Springer, Berlin, Germany, 2007.

[33] P. Venkataraman, Applied Optimization with MATLAB Programming, John Wiley \& Sons, Hoboken, NJ, USA, 2009.

[34] M. Hoeft and J. Rak, "How to provide fair service for V2I communications in VANETs?" Ad Hoc Networks, vol. 37, pp. 283-294, 2016.

[35] C. Mun, J. Choi, Y. Kim, M. Baek, G. Seo, and K. Ko, "Cell planning and deployment for IEEE 802.11pWAVE network," IEEE Intelligent Transportation Systems Magazine, vol. 7, no. 4, pp. 49-57, 2015. 


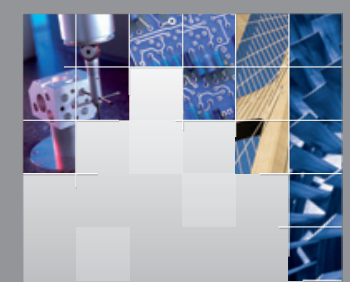

\section{Enfincering}
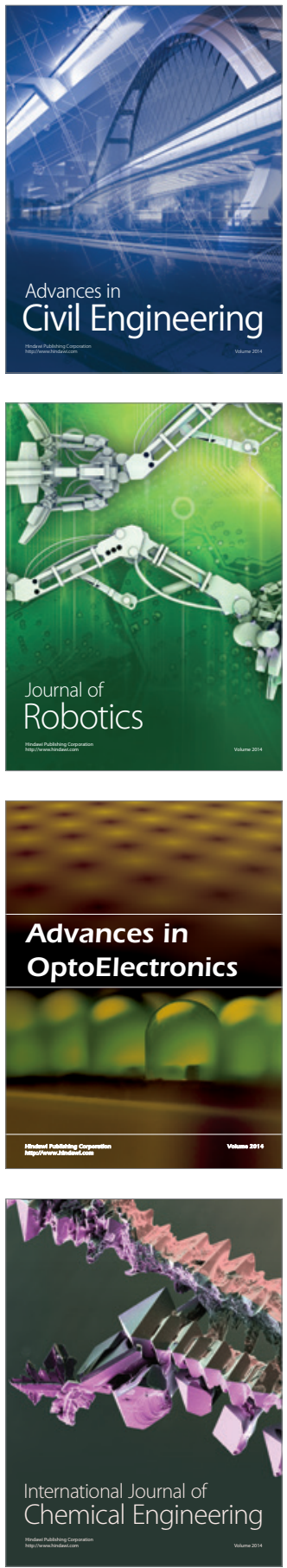

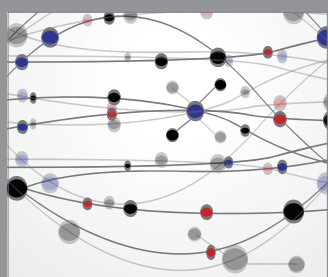

The Scientific World Journal

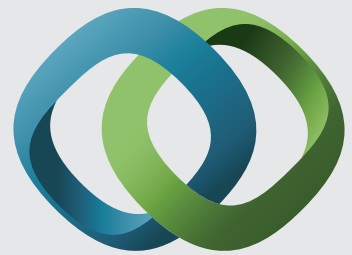

\section{Hindawi}

Submit your manuscripts at

http://www.hindawi.com
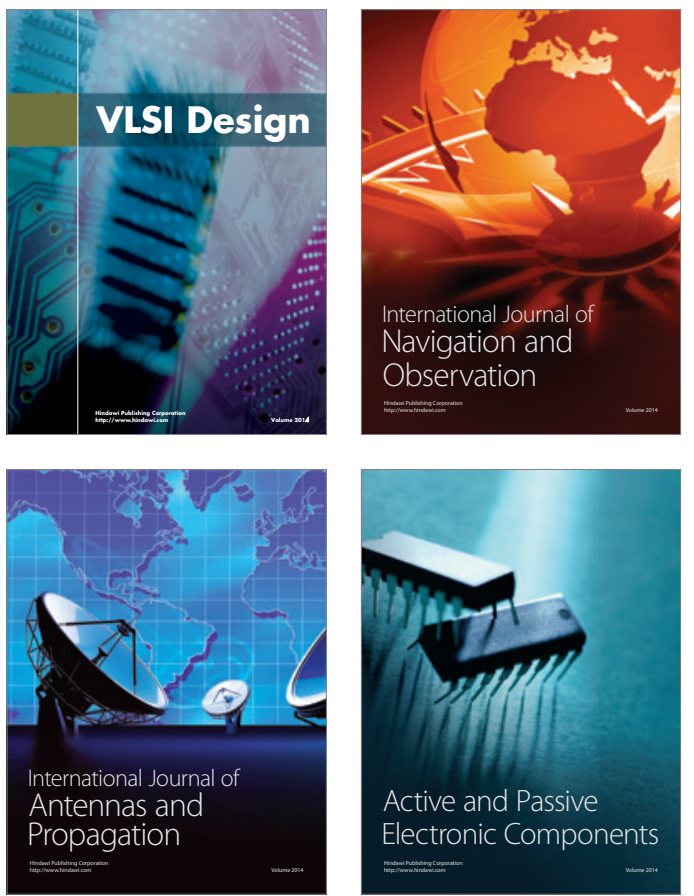
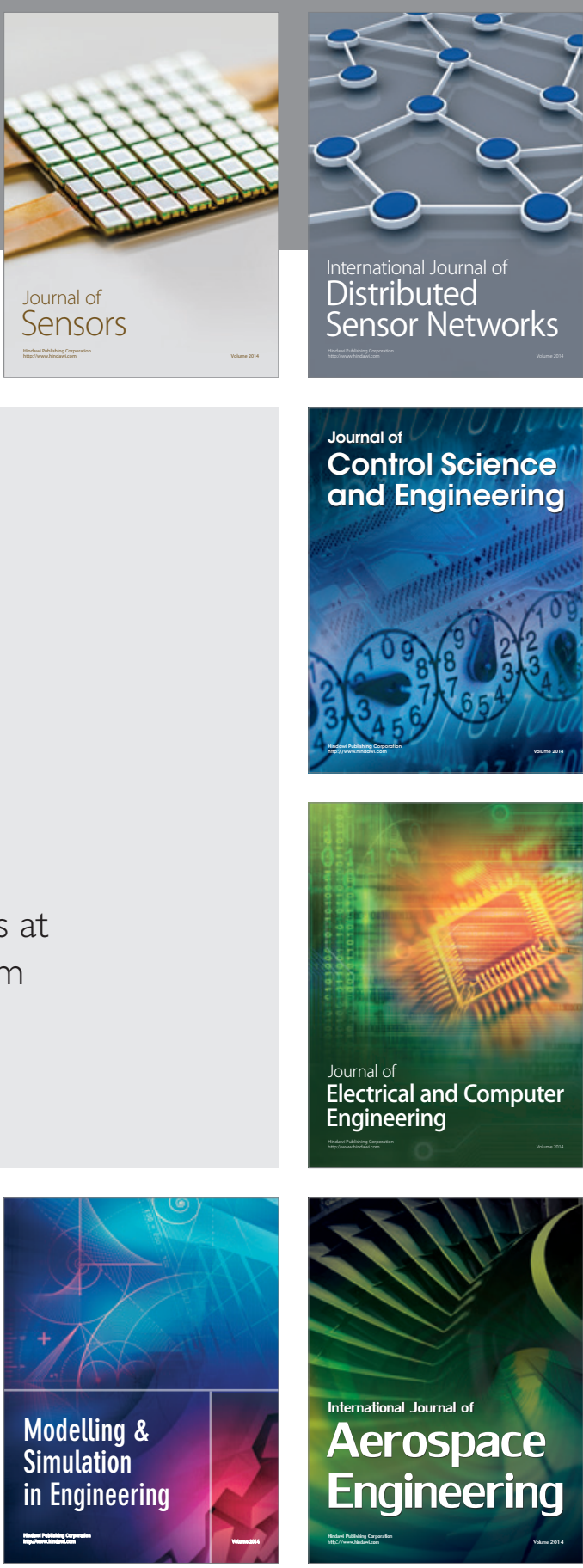

International Journal of

Distributed

Sensor Networks

Journal of

Control Science

and Engineering
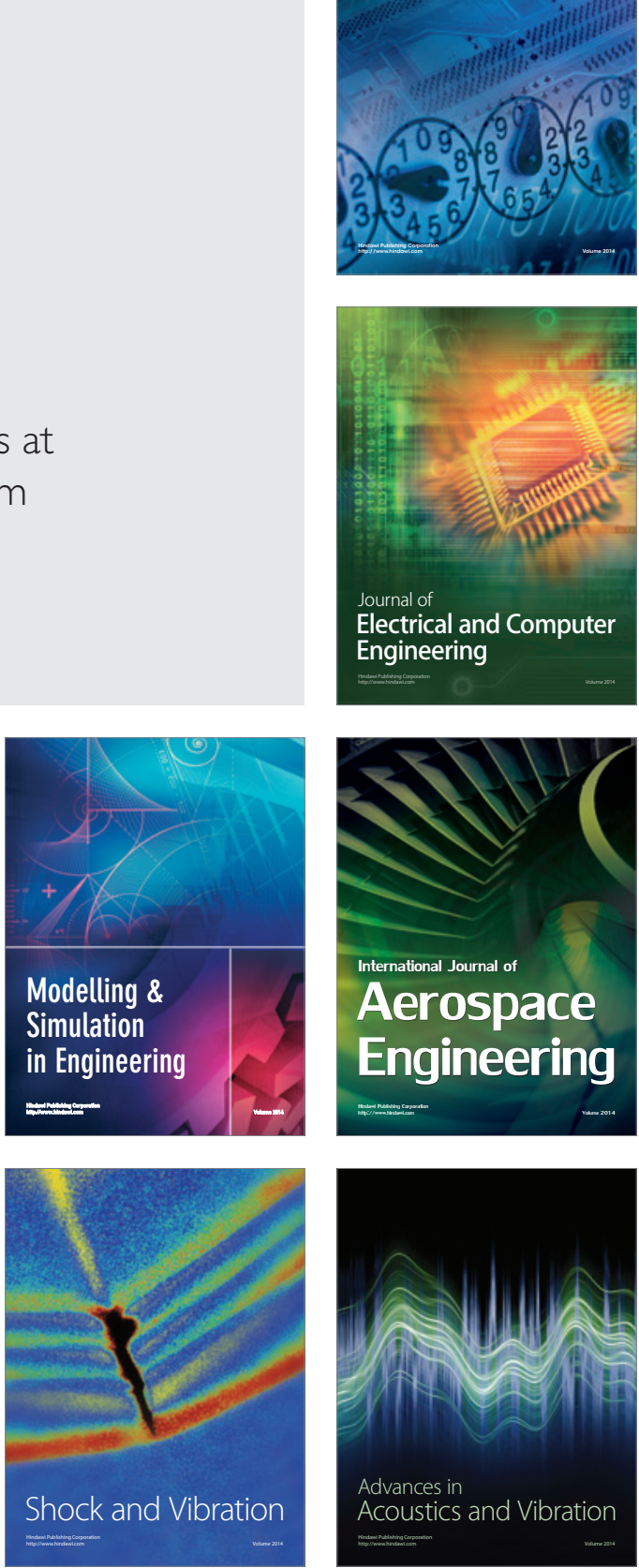